\title{
Pancreatic Adenocarcinoma: Role of the Site-Specific of Metastases
}

\author{
Vitorino Modesto dos Santos ${ }^{1^{*}}$ and Lister Arruda Modesto dos Santos ${ }^{2}$ \\ ${ }^{1}$ Department of Internal Medicine, Armed Forces Hospital, Catholic University, Brasilia-DF, Brazil \\ ${ }^{2}$ Department of General Surgery, State Worker's Hospital, São Paulo-SP, Brazil
}

*Corresponding author: Vitorino Modesto dos Santos, Department of Internal Medicine, Armed Forces Hospital, Catholic University, Estrada do Contorno do Bosque s/n, Cruzeiro Novo, CEP 70.658-900, Brasília-DF, Brazil, Tel: +556-139-662-103, Fax: 556-132-331-599, E-mail: vitorinomodesto@gmail.com

\begin{abstract}
Pancreatic adenocarcinoma is a severe malignant condition with a median survival lower than one year, and a five-year survival rate of about $30 \%$, including tumors resected at early stage. Recurrences within two years occur in up to $80 \%$ of the resected tumors. More than two thirds of advanced pancreatic adenocarcinomas have single site of metastases, mainly affecting the liver, lungs, bones and brain, by decreasing order of frequency. The studies herein commented suggest that patients with lung-only metastases or with isolated distant lymph node implants have better outcomes than those with liver-only metastases. The physiopathological mechanisms are not well known; moreover one should consider the role of Trousseau's syndrome on estimated survival time. This paraneoplastic syndrome is characterized by hypercoagulability causing arterial and venous thromboses.
\end{abstract}

\section{Keywords}

Metastases, Pancreatic adenocarcinoma, Trousseau's syndrome

\section{Introduction}

Recently, we have read very interesting papers about epidemiological features and the site-specific prognostic role played by metastases of Pancreatic Adenocarcinoma (PAC) [1-3]. PAC is a very ominous malignancy with a median survival less than 1 year for metastatic disease and about 2 years for resect disease, an overall 5 -year survival rate around $5 \%$ to $8 \%$ for metastatic disease and about $30 \%$ in case of early diagnosis and early surgical resection $[1,2]$. Recurrences may occur within two years in up to $80 \%$ of the patients who underwent tumor resections [1]. Late recurrences predominate among people with exclusive lung metastases, but ar- terial and venous invasions are similar to those with exclusive liver metastases [1,2]. Among 13233 patients with advanced PCA, $66.3 \%$ had single site of metastases; and the main affected organs were: Liver, lungs, bones and brain, by decreasing order of frequency [3].

In 2013, Murinello, et al. reported the case of a 58-year-old man with venous thrombosis in the upper and lower limbs and lung embolism related to unsuspected PAC and hepatic metastases [4]. The patient was a tobacco smoker, with a mild alcohol intake. There was no fever, sweating, weight loss, respiratory, digestive, urinary or other symptoms [4]. Laboratory tests revealed elevated levels of GGT, ALP, LDH, amylase and lipase, the tumor markers CA 19.9 and CEA were within normal range. Imaging studies showed an infiltrative mass in the pancreatic uncinate process involving the superior mesenteric vessels, embolism in the upper and inferior lobes of the right lung; and nodules compatible with hepatic implants. Further aspiration biopsy of the liver and pancreas confirmed PAC with metastases, the patient evolved to death due to ischemic stroke, necropsy studies were not done [4]. The authors focused on the Trousseau's syndrome, a thrombotic diathesis associated with cancer of pancreas, stomach, colon and rectum, lung, breast, ovary or prostate, producing mucin [4].

Reviewing our old files, we found the case of a 67-year-old woman admitted with chronic intestinal constipation, asthenia, anorexia and weight loss, and recent progressive jaundice. Physical examination showed anicteric and malnourished patient, with a painful hard mass on the epigastrium. There was no fe-

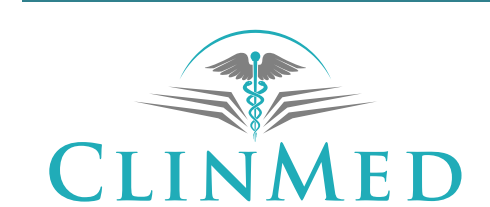

INTERNATIONAL LIBRARY 


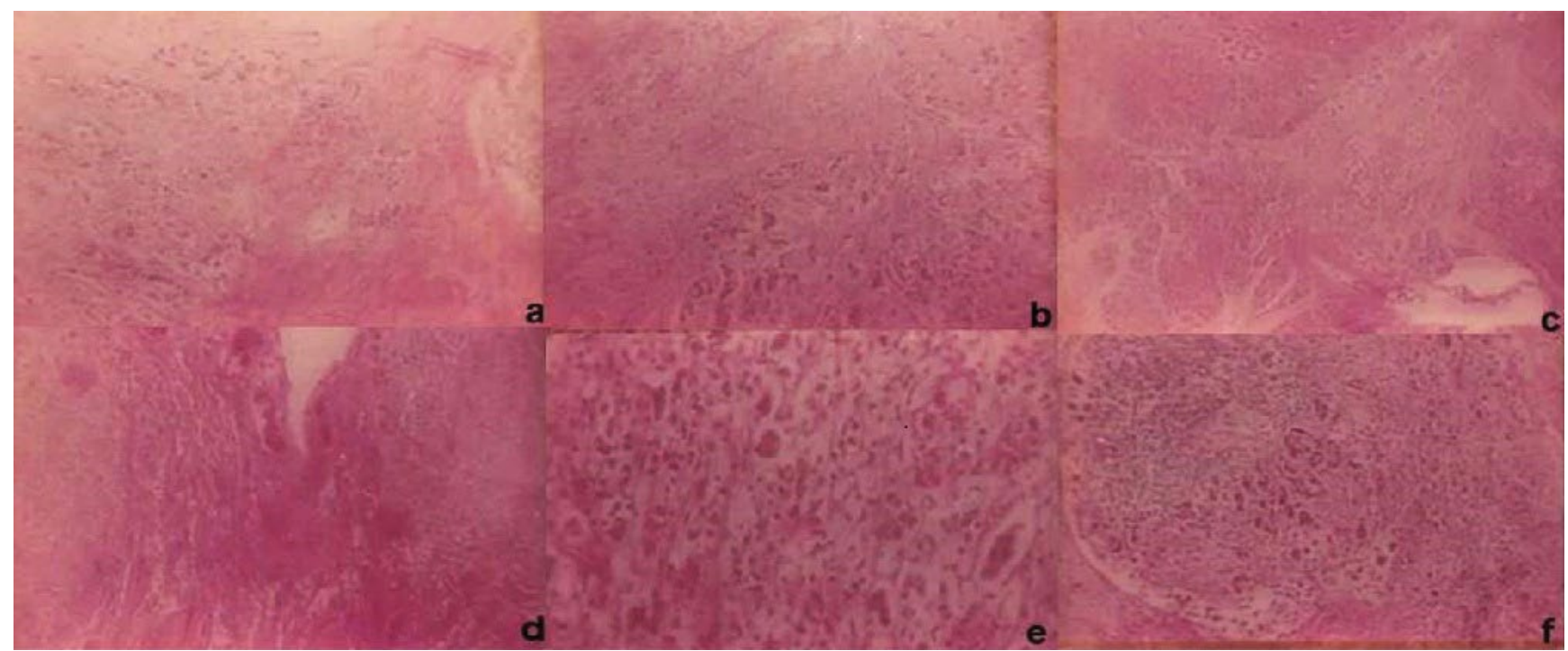

Figure 1: Photomicrography features of necropsy specimens (HE $\times 100$ magnifications) a) Pancreatic parenchyma permeated by neoplastic glands and some signet ring cells; b,c) Aspect of duodenum with muscular and submucosa layers involved by the tumor; d,e) Details of the pulmonary area affected by metastatic involvement of the pancreatic tumor; f) Appearance of a lymph node with implants of neoplastic cells from the pancreatic cancer.

ver and pulmonary or lymph node changes. The mass had no continuity with the liver, which was at $6 \mathrm{~cm}$ below the right costal border with a blunt border; and the spleen was unremarkable. Routine tests showed anemia, leukocytosis, elevation of conjugated bilirubin and of canalicular enzymes; and the levels of transaminases were normal. Serum determinations of tumor biomarkers revealed elevated levels of CEA and CA 19-9. Imaging studies of the digestive tract showed abnormality in the area of the ampulla of Vater; in the head of the pancreas there was a mass causing bile duct dilatation, hepatic nodules, in addition to numerous peripancreatic lymph nodes with significant increase of volume. Chest radiography revealed a nodule of imprecise limits in the lower lobe of the right lung. Complete clinical investigation was not carried out due to sudden death. Necropsy study was authorized and diagnostic challenges were solved, similarly to the findings of Morinello, et al. above commented, mesenteric vessels were affected. She had synchronous lung and liver metastases and died before any treatment due to pulmonary thromboembolism associated with the Trousseau's syndrome. Histopathological analysis of necropsy samples was consistent with PAC and hepatic, pulmonary and lymph node metastases (Figure 1). The immune histochemistry (not shown) pattern S100P+/MUC5AC+/CK17+/pVHL- was an additional finding to confirm the primary pancreatic origin of the disseminated ductal adenocarcinoma.

Data from the case studies herein commented suggest that patients with lung-only PDC metastases or with isolated distant lymph node implants have better outcomes than those with liver-only metastases [1-3]. However, the physiopathology of involved mechanisms should be better cleared, moreover one should consider the role of Trousseau's syndrome on estimated survival time either in advanced-stage or in early phase cancer.

\section{Financial Disclosure}

There was no grant support for this study.

\section{Disclosure of Potential Conflicts of Interest}

The authors had full freedom of manuscript preparation and there were no potential conflicts of interest.

\section{Acknowledgement}

The authors would like to thank Almir José Batista, the official photographer of the Armed Forces Hospital, in Brasilia-DF, Brazil for the images that illustrate the present case study.

\section{References}

1. Decoster C, Gilabert M, Autret A, Turrini O, Oziel Taieb S, et al. (2016) Heterogeneity of metastatic pancreatic adenocarcinoma: Lung metastasis show better prognosis than liver metastasis- a case control study. Oncotarget 7: 45649-45655.

2. Downs Canner S, Zenati M, Boone BA, Varley PR, Steve J, et al. (2015) The indolent nature of pulmonary metastases from ductal adenocarcinoma of the pancreas. J Surg Oncol 112: $80-85$.

3. Oweira H, Petrausch U, Helbling D, Schmidt Mannhart M, Mehrabi A, et al. (2017) Prognostic value of site-specific metastases in pancreatic adenocarcinoma: A surveillance epidemiology and end results database analysis. World $\mathrm{J}$ Gastroenterol 23: 1872-1880.

4. Murinello A, Guedes P, Rocha G, Serrano A, Figueiredo A, et al. (2013) Trousseau's syndrome due to asymptomatic pancreatic adenocarcinoma. GE J Port Gastrenterol 20: 172-176. 